\title{
Increased Abdominal Adiposity Exacerbates Ex-Vivo Cardiac Reperfusion Injury through Augmented Mitochondrial Oxidative Stress
}

Damien Vitiello $^{1-4 *}$, Evangelia Mourmoura ${ }^{1,2}$, Karine Couturier ${ }^{1,2}$, Patrick Faure ${ }^{5}$, Herve Dubouchaud ${ }^{1,2}$, Corinne Malpuech-Brugere ${ }^{6}$, Kasra Azarnoush $^{6,7}$ and Luc Demaison ${ }^{1,2,6}$

${ }^{1}$ Laboratoire de Bioénergétique Fondamentale et Appliquée, INSERM U1055, Grenoble cedex 09, F-38041, France

${ }^{2}$ Université Joseph Fourier, Laboratoire de Bioénergétique Fondamentale et Appliquée, Grenoble cedex 09, F-38041, France

${ }^{3}$ Institut de Recherche biomédicale et d'Épidemiologie du Sport (IRMES), Institut National du Sport de l'Expertise et de la Performance (INSEP), Paris, France.

${ }^{4}$ Université Paris Descartes, Sorbonne Paris Cité, EA 7329, Paris, France

${ }^{5}$ Institut de Biologie et de Pathologie, DBTP, CHU de Grenoble, F-38043 Grenoble cedex 09, France

${ }^{6}$ Unité de Nutrition Humaine, INRA, UMR 1019, Clermont Université, Université d'Auvergne, BP 10448, F-63000 Clermont-Ferrand, France

${ }^{7}$ Clermont-Ferrand University Hospital, Heart Surgery Department, G. Montpied Hospital, F-63000 Clermont-Ferrand, France

\begin{abstract}
Compared to the lean Lou/C rat, the Wistar rat develops increased body weight and abdominal adiposity (IAA). The aim of this study was to compare the functional response to cardiac ischemia/reperfusion of 3-month old Wistar rats with age-matched Lou/C rats, and to explain the differences with regards to mitochondrial hydrogen-peroxide release $\left(\mathrm{mH}_{2} \mathrm{O}_{2} \mathrm{r}\right)$. Langendorff-perfused hearts of Lou/C and Wistar rats were subjected to ischemia ( $\left.25 \mathrm{~min}\right)$ followed by reperfusion (30 min). Cardiac function was monitored throughout the experiment. Mitochondria were extracted before and after ischemia, and their oxidative capacities, $\mathrm{mH}_{2} \mathrm{O}_{2} \mathrm{r}$, and activity of the respiratory-chain complex were measured. The IAA of Wistar rats was associated with slight glucose intolerance and noticeable functional abnormalities of the myocardium during post-ischemic reperfusion. Cardio-toxicity was related to maintenance of the activity of respiratory-chain complex II and increased $\mathrm{mH}_{2} \mathrm{O}_{2} \mathrm{r}$, whereas cardio-protection in lean Lou/C rats occurred through reduced complex-II activity and $\mathrm{mH}_{2} \mathrm{O}_{2}$ r. In conclusion, IAA and/or systemic glucose intolerance maintained complex-II activity during post-ischemic reperfusion, thus increasing $\mathrm{mH}_{2} \mathrm{O}_{2} \mathrm{r}$ through reverse electron flux and inducing significantly increased cardio-toxicity. Inhibitors of respiratory complex II could thus help protect the heart against ischemia/reperfusion in obese individuals.
\end{abstract}

Keywords: Abdominal obesity; Oxidative stress; Cardiac ischemia; Energy metabolism; Glucose tolerance

\section{Introduction}

Obesity exacerbates the incidence of coronary heart disease [1] but seems to have a contradictory effect on cardiac susceptibility to ischemic damage (CSI). Several models of obesity have been developed regarding energy-dense food and peri-natal over-nutrition in normal animals, as well as genetic models of hyperphagy. The effects of cardiac ischemia/reperfusion differ according to the chosen model.

Sweetened condensed milk (SCM) and high-fat (HF) diets are the main energy-dense food diets tested. With SCM, CSI decreases $[2,3]$ whereas, with HF diets, similar to so-called Western diets rich in saturated fats, CSI increases [4-9].

Both peri-natal over-nutrition $(\mathrm{ON})$ and genetically modified rodents with a hyperphagic behaviour have been found to have increased calorie intake, but when allowed to eat a normal diet, ON rodents have increased CSI [10-12]. The diabetic Zucker fatty rat, a genetic model of hyperphagy and hyperglycaemia caused by a Westerntype diet, displays decreased CSI until the age of 6 months $[13,14]$ but then has increased CSI thereafter [15].

In humans, obesity can result from an increased intake of equilibrated food and does not automatically induce hyperglycaemia. It is thus important to use a model that assembles these characteristics to mimic this situation. The Lou/C rat, an animal selected from the Wistar strain, displays spontaneous caloric restriction $[16,17]$, which appears to be related to reduced expression of orexigenic neuropeptides [18]. This rat phenotype can maintain insulin sensitivity throughout its lifespan [19], and displays a resistance to age- and diet-induced obesity compared to Wistar rats $[20,21]$. The Lou/C rat has lower plasma leptin and higher adiponectin concentrations [22,23], reflecting lower visceral fat deposition [19,21,24-26]. Preferential channeling of lipid nutrients toward utilization rather than to storage has been proposed to explain this anti-obese response [21,25]. Lacraz et al. [27] have suggested that augmented lipid oxidation, specifically within the liver, could explain this lifelong maintained insulin sensitivity. Comparison of the characteristics between lean Lou/C and Wistar rats are of particular interest when evaluating the effects of increased body and abdominal fat masses on CSI in the context of a balanced diet.

Obesity has often been associated with oxidative stress [28], which is particularly relevant when ischemia/reperfusion is studied. The main disorders encountered in myocardial infarction occur during reperfusion and are related to oxidative stress. The adenine nucleotide dephosphorylation that occurs during ischemia [29] leads to the production of two types of reactive oxygen species (ROS) during reperfusion. The first ROS becomes highly elevated, but only for a

*Corresponding author: D. Vitiello, Institut de Recherche biomédicale et d'Épidemiologie du Sport (IRMES), Institut National du Sport de l'Expertise et de la Performance (INSEP), Paris, France. Université Paris Descartes, Sorbonne Paris Cité, EA 7329, Paris, France, Tel: +33-156-561200; E-mail: damien.vitiello@parisdescartes.fr

Received September 21, 2015; Accepted October 08, 2015; Published October 12, 2015

Citation: Vitiello D, Mourmoura E, Couturier K, Faure P, Dubouchaud H, et al (2015) Increased Abdominal Adiposity Exacerbates Ex-Vivo Cardiac Reperfusion Injury through Augmented Mitochondrial Oxidative Stress. J Diabetes Metab 6: 614. doi:10.4172/2155-6156.1000614

Copyright: ( 2015 Vitiello D, et al. This is an open-access article distributed unde the terms of the Creative Commons Attribution License, which permits unrestricted use, distribution, and reproduction in any medium, provided the original author and source are credited. 
short duration at the beginning of reperfusion [30]; it is caused by oxidation of hypoxanthine by xanthine oxidase [31]. The second type of ROS becomes less increased, but for a long duration: it is caused by intracellular acidosis [32], which leads to intracellular calcium accumulation [33].

Abnormalities in electrolyte homeostasis occur in obese individuals [34]. In the reperfused myocardium, excess calcium invades the mitochondria [35] and triggers noticeable ROS production [36]. ROS inhibit the aconitase enzyme within the Krebs cycle [37], which blocks ATP synthesis and triggers the functional abnormalities that characterize reperfusion [38]. Obesity may modulate mitochondrial ROS production and the recovery of cardiac functional activity during reperfusion.

This study aimed to determine whether increased abdominal adiposity (IAA) during the early phase of systemic glucose intolerance due to augmented intake of a low-fat diet equilibrated in lipids, can alter the recovery of mechanical function during post-ischemic reperfusion. We also wanted to determine if mitochondrial oxidative stress was involved, and to determine the reasons for the changes in ROS production. For this purpose, we compared the response to ischemia/ reperfusion of two phenotypes of 3-month old Wistar rats: one that becomes obese at an advanced age (standard Wistar) and the other that remains lean (Lou/C). To highlight the mechanisms involved, several features of ex-vivo perfused hearts were determined (coronary flow, oxygen consumption, cardiac metabolic efficiency, glycolytic rate). Mitochondria were extracted from pre- and post-ischemic hearts and their function (oxidative phosphorylation, $\mathrm{H}_{2} \mathrm{O}_{2}$ release) was estimated. Finally, mitochondrial oxidative stress and the activity of respiratorychain complexes were measured in the reperfused hearts.

\section{Materials and Methods}

\section{Animals and diet}

The experiments followed the European recommendation guidelines for the use of laboratory animals and were approved by the local ethics review board (Cometh, authorization number: 152_LBFAU1055-LD-03).

Twenty-two 3-month-old male Wistar rats and 22 age-matched Lou/C rats were housed at two per cage in an animal facility, with controlled temperature, dark/light cycles and hygrometry. All animals were kept under sedentary conditions. They were fed standard commercial pellets (A04, Safe, Gannat, France) ad libitum with free access to water. The diet contained $3.1 \%$ lipids, $16.1 \%$ proteins and $60 \%$ cellulose, and was equilibrated in saturated (24\%), monounsaturated (23\%), n-6 polyunsaturated (48\%) and n-3 polyunsaturated $(4.5 \%)$ fatty acids. The animals of each phenotype were randomly divided into two groups to evaluate ex-vivo cardiac function during ischemia/ reperfusion $(n=10)$ and mitochondrial function $(n=12$, i.e. 6 preischemic hearts and 6 post-ischemic hearts).

Animal size was estimated by determining the length from the insertion of the collarbones on the sternum to the penis. Animal density was determined by the ratio between the animal's weight and length.

\section{Oral glucose tolerance test}

An oral glucose tolerance test was performed 10 days before sacrifice. Food was removed from rats $18 \mathrm{~h}$ before they were given an oral dose of glucose ( $1 \mathrm{~g}$ glucose $/ \mathrm{kg}$ body weight, between 08.00 and 10.00 a.m.). Blood samples were collected from the tail vain in heparinized tubes immediately before glucose administration to determine basal glucose and insulin values, and also at 25,60 and $180 \mathrm{~min}$ after administration.

Glucose values were determined with a glucose analyzer (ACCUCHECK Active, Softclix). After centrifugation $\left(3000 \mathrm{~g}, 7 \mathrm{~min}, 4^{\circ} \mathrm{C}\right)$ plasma samples were stored at $-20^{\circ} \mathrm{C}$ until insulin was determined using a radioimmunoassay kit (SRI-13K, Millipore, Molheim, France). The total area under the curve for glucose and insulin was then calculated to evaluate glucose tolerance.

\section{Isolated heart perfusion}

The animals were anesthetized with sodium pentobarbital $(50 \mathrm{mg} /$ $\mathrm{kg}$ ) and were then heparinized $(1000 \mathrm{IU} / \mathrm{kg})$ through the saphenous vein. After rapid excision of the heart, the aorta was cannulated using the Langendorff technique to allow non-recirculating coronary perfusion at a constant pressure $(60 \mathrm{mmHg})$ and at $37^{\circ} \mathrm{C}$. The modified Krebs-Henseleit perfusion buffer contained: $\mathrm{NaCl}(119 \mathrm{mM}), \mathrm{MgSO}$ (1.2 mM), $\mathrm{NaHCO}_{3}(25 \mathrm{mM}), \mathrm{KCl}(4.8 \mathrm{mM}), \mathrm{CaCl}_{2}(2.5 \mathrm{mM})$ and glucose $(11 \mathrm{mM})$, and was equilibrated with $95 \% \mathrm{O}_{2}-5 \% \mathrm{CO}_{2}$. A latex balloon linked to a pressure probe was inserted into the left ventricle to determine diastolic and systolic pressures. Baseline diastolic pressure was set at $\sim 10 \mathrm{mmHg}$. Coronary flow was estimated by weighing samples of effluent collected for $30 \mathrm{~s}$. The pulmonary artery was cannulated for direct coronary-sinus sampling of the coronary effluent.

Arterial and venous oxygen contents were measured by anaerobic collection of effluent in hermetically closed capillary tubing. The oxygen concentration was determined with a blood-gas analyzer (Radiometer ${ }^{\mathrm{TM}} \mathrm{ABL}$ 700, Brønshøj, Denmark). The left ventricular developed pressure (LVDP) was calculated as the difference between systolic and diastolic pressures. The rate pressure product (RPP) was the product between heart rate and LVDP. Oxygen consumption was calculated as the difference between the product of arterio-venous oxygen content and coronary flow. Since the blood-gas analyzer gave oxygen concentrations in $\mathrm{kPa}$, the difference in arterio-venous oxygen content was calculated assuming that $1 \mathrm{kPa}=10 \mathrm{nmoles}$ of oxygen per $\mathrm{mL}$ [39]. Cardiac metabolic efficiency was estimated as the ratio between RPP and oxygen consumption and was expressed in $\mathrm{mHg} /$ umole.

\section{Ischemia-reperfusion protocol and sampling}

Ten hearts of each phenotype were perfused for $30 \mathrm{~min}$ to establish stabilized baseline performance before inducing total global normothermic ischemia for $25 \mathrm{~min}$ and reperfusion for $30 \mathrm{~min}$. At the end of the reperfusion period, the hearts were freeze-clamped in liquid nitrogen and stored at $-80^{\circ} \mathrm{C}$ until determination of heart dry weight and enzymatic activity. In another set of experiments, six hearts of each phenotype were perfused under baseline conditions for $30 \mathrm{~min}$, and six other hearts were subjected to normoxia $(30 \mathrm{~min})$ followed by ischemia $(25 \mathrm{~min})$ and reperfusion $(1 \mathrm{~min})$. At the end of each of these two protocols, cardiac mitochondria were immediately extracted.

\section{Preparation of mitochondria}

After perfusion, the hearts were minced with scissors in a cold isolation buffer composed of sucrose $(150 \mathrm{mM}), \mathrm{KCl}(75 \mathrm{mM})$, Tris$\mathrm{HCl}(50 \mathrm{mM}), \mathrm{KH}_{2} \mathrm{PO}_{4}(1 \mathrm{mM}), \mathrm{MgCl}_{2}(5 \mathrm{mM})$ and EGTA $(1 \mathrm{mM})$, at $\mathrm{pH} 7.4$, with fatty-acid-free serum albumin $(0.2 \%)$. The pieces of myocardium were rinsed on a filter and placed in a Potter-Elvehjem tissue grinder that contained $15 \mathrm{~mL}$ of isolation buffer. Protease (subtilisin $0.02 \%$ ) was added to digest the myofibrils for $1 \mathrm{~min}$ at $4^{\circ} \mathrm{C}$ and the whole preparation was then homogenized with the grinder (at $300 \mathrm{rpm}$, for 3-4 transitions). Subtilisin action was stopped by adding 
Citation: Vitiello D, Mourmoura E, Couturier K, Faure P, Dubouchaud H, et al. (2015) Increased Abdominal Adiposity Exacerbates Ex-Vivo Cardiac Reperfusion Injury through Augmented Mitochondrial Oxidative Stress. J Diabetes Metab 6: 614. doi:10.4172/2155-6156.1000614

Page 3 of 10

the isolation buffer $(30 \mathrm{~mL})$. The homogenate was then centrifuged $\left(800 \mathrm{~g}, 10 \mathrm{~min}, 4^{\circ} \mathrm{C}\right)$, and the resulting supernatant was collected and filtered. Mitochondria were then washed via two series of centrifugation $\left(8000 \mathrm{~g}, 10 \mathrm{~min}, 4^{\circ} \mathrm{C}\right)$. The last pellet of mitochondria was re-suspended in sucrose $250 \mathrm{mM}$ Tris- $\mathrm{HCl} 10 \mathrm{mM}$, EGTA $1 \mathrm{mM}$, at $\mathrm{pH}$ 7.4, at an approximate concentration of $20 \mathrm{mg} / \mathrm{mL}$.

\section{Mitochondrial respiration and $\mathrm{H}_{2} \mathrm{O}_{2}$ release $\left(\mathrm{mH}_{2} \mathrm{O}_{2} \mathbf{r}\right)$}

Oxidative phosphorylation and $\mathrm{mH}_{2} \mathrm{O}_{2} \mathrm{r}$ were determined as previously described [40]. Two different respiration buffers with different calcium concentrations $(0$ or $10 \mu \mathrm{M})$ were used. They contained $\mathrm{KCl} 125 \mathrm{mM}$, Tris- $\mathrm{HCl} 20 \mathrm{mM}, \mathrm{KH}_{2} \mathrm{PO}_{4} 3 \mathrm{mM}$, EDTA plus $\mathrm{CaCl}_{2}$ (1 plus 0 , or 0.005 plus 0.01 ), at $\mathrm{pH} 7.2$, plus fatty-acid-free bovine serum albumin $(0.15 \%)$. All measurements were performed on mitochondria $(0.2 \mathrm{mg}$ mitochondrial protein $/ \mathrm{mL})$ incubated with freshly prepared pyruvate $(5.5 \mathrm{mM}) /$ malate $(2.5 \mathrm{mM})$ or DLpalmitoylcarnitine $(50 \mu \mathrm{M})$ /malate $(2.5 \mathrm{mM})$, or a combination of these three substrates. State-III and state-IV respiration rates (StIII and StIV), as well as the respiratory control ratio (RCR), were determined as previously described [40]. The $\mathrm{mH}_{2} \mathrm{O}_{2} \mathrm{r}$ was determined during stateII respiration.

\section{Western blotting}

Protein levels in the monocarboxylate transporter-1 (MCT1) and sodium/proton exchanger-1 (NHE1) were determined on the membrane fraction of the frozen samples from the reperfused left ventricle using Western blotting analysis. Samples (50-100 mg) were homogenized in $210 \mathrm{mM}$ sucrose, $2 \mathrm{mM}$ EGTA, $40 \mathrm{mM} \mathrm{NaCl}, 30 \mathrm{mM}$ HEPES, at $\mathrm{pH} 7.4$, and supplemented with a $0.15 \%$ protease inhibitor cocktail (Sigma P-8340). The membrane fraction was then prepared according to Dubouchaud et al. [41]. The proteins were assayed and $100-\mu \mathrm{g}$ molecular-weight markers, were separated out by $8-16 \%$ SDSpolyacrylamide gel electrophoresis and transferred, by electro-blotting, onto nitrocellulose membranes. The membranes were incubated for $2 \mathrm{~h}$ in blocking buffer $(150 \mathrm{mM} \mathrm{NaCl}, 5 \%$ non-fat dried milk, $0.1 \%$ Tween 20, $10 \mathrm{mM} \mathrm{NaH} \mathrm{PO}_{4}$, at $\mathrm{pH}$ 7.4). They were cut into two parts between the 50 - and $75-\mathrm{KDa}$ molecular-weight markers to separate out MCT-1 (43 KDa) and NHE-1 (92 KDa). Each part was then incubated overnight at $4^{\circ} \mathrm{C}$ with the appropriate primary antibodies. The antiMCT1 antibody was a generous gift from Prof. Brooks (University of California, Berkeley). The anti-NHE1 antibody was obtained from BD Biosciences (ref\# 611774). The membranes were washed in 150 $\mathrm{mM} \mathrm{NaCl}, 10 \mathrm{mM} \mathrm{NaH}_{2} \mathrm{PO}_{4}$, at $\mathrm{pH} 7.4$, and then incubated for $1 \mathrm{~h}$ with a horseradish peroxidase-linked secondary antibody (Amersham Biosciences, Orsay, France).

Protein contents were detected by enhanced chemiluminescence, and band densities were quantified using Scion Image software (Scion Corporation). To ensure consistency between experiments, a small aliquot of the same sample was systematically loaded on every gel and the generated signal was used as 1 arbitrary unit to express the results. The quantification of MCT1 and NHE1 from the same deposit allowed us to calculate the ratio between these two proteins.

\section{Biochemical analyses}

Lactate and pyruvate in the coronary effluents from each heart were determined according to Bergmeyer [42]. Glycolytic flux was calculated as the sum of lactate and pyruvate released into the coronary effluent. Citrate-synthase activity was determined [43] in myocardium to estimate mitochondrial density. Aconitase and fumarase activities were determined in homogenates of the reperfused myocardium, according to the method of Gardner et al. [44]. Respiratory-chain complex activities were also measured in homogenates from the reperfused myocardium, as previously described [40]. Proteins were measured using the bicinchoninic acid method (Thermo Scientific, Rockford, IL) [45].

\section{Determination of cardiac dry weight}

The wet weight of the whole heart was evaluated and a piece of myocardium was placed in an oven at $100^{\circ} \mathrm{C}$ for $24 \mathrm{~h}$. Weight determination was carried out before and after drying to provide the percentage dry weight. Cardiac dry weight was estimated by multiplying the wet weight by the percentage dry weight.

\section{Statistical analyses}

The results are presented as their means \pm SEMs. Data on general animal morphology, baseline normoxic cardiac function and mitochondrial oxidative stress during reperfusion were contrasted between the phenotypes by one-way ANOVA. Mitochondrial respiration and $\mathrm{mH}_{2} \mathrm{O}_{2} \mathrm{r}$ were analyzed using three-way ANOVA with three fixed factors (phenotype, ischemia/reperfusion and calcium concentration). Time-evolutions of glycaemia and insulinemia, after the glucose loading, were analyzed with repeated-measure ANOVA using the phenotype as an external factor and the time at post-glucose loading as the internal factor. Measurements made during ischemiareperfusion (diastolic pressure, developed pressure, heart rate, RPP, oxygen consumption, lactate, pyruvate, metabolic efficiency) were analyzed with a repeated-measure ANOVA to test the effect of phenotype (external factor), perfusion times (internal factor) and their interactions. When required, group means were contrasted using Fisher's least-significant difference test. A probability of $p<0.05$ was considered statistically significant.

\section{Results}

\section{General data}

The Wistar rats had higher food consumption than their Lou/C counterparts $(63.3 \pm 1.9$ vs. $36.4 \pm 1.4 \mathrm{Kcal} / \mathrm{d} ;+74 \%, p<0.001)$. Their body weights and densities were also higher compared to Lou/C rats (323 \pm 5 vs. $188 \pm 11 \mathrm{~g},+72 \%, p<0.001$ and $22.6 \pm 0.3$ vs. $15.6 \pm 0.7$ $\mathrm{g} / \mathrm{cm},+45 \%, p<0.001$, respectively). This was partly due to increased abdominal adiposity $(3.7 \pm 0.2$ vs. $2.1 \pm 0.1 \mathrm{~g} / 100$ g of body weight, + $76 \%, p<0.001)$. The leg-muscle mass of Wistar rats was lower $(642 \pm$ 7 vs. $727 \pm 5 \mathrm{mg} / 100 \mathrm{~g}$ of body weight, $-12 \%, p<0.001)$. Similarly, the relative heart weight of Wistar rats was significantly lower $(63 \pm 2 v s$. $77 \pm 2 \mathrm{mg}$ of dry myocardium/100 g of body weight, $-18 \%$, $p<0.001$ ).

\section{Oral glucose tolerance tests}

Fasting glycaemia and insulinemia (Figure 1A and C) were not altered by rat phenotype. In the glucose challenge, the area under the curve for glycaemia (Figure 1B) was slightly but significantly higher in the Wistar strain $(+4 \%)$. This was caused by an increased plasmaglucose concentration $(+7 \%)$ at 25 min post-glucose-loading, which was associated with augmented insulinemia $(+70 \%)$.

\section{Cardiac function under pre-ischemic conditions}

To evaluate global cardiac function, the parameters of cardiac mechanical function (systolic, diastolic and LVDP as well as RPP) were not normalized to heart weight (Table 1). Systolic, diastolic and LVDP were similar between the two phenotypes but, because of lower heart rate $(-13 \%)$, RPP was reduced $(-12 \%)$ in the Wistar phenotype. The hearts of Wistar rats released more lactate $(+36 \%)$, and the ratio 
Citation: Vitiello D, Mourmoura E, Couturier K, Faure P, Dubouchaud H, et al. (2015) Increased Abdominal Adiposity Exacerbates Ex-Vivo Cardiac Reperfusion Injury through Augmented Mitochondrial Oxidative Stress. J Diabetes Metab 6: 614. doi:10.4172/2155-6156.1000614
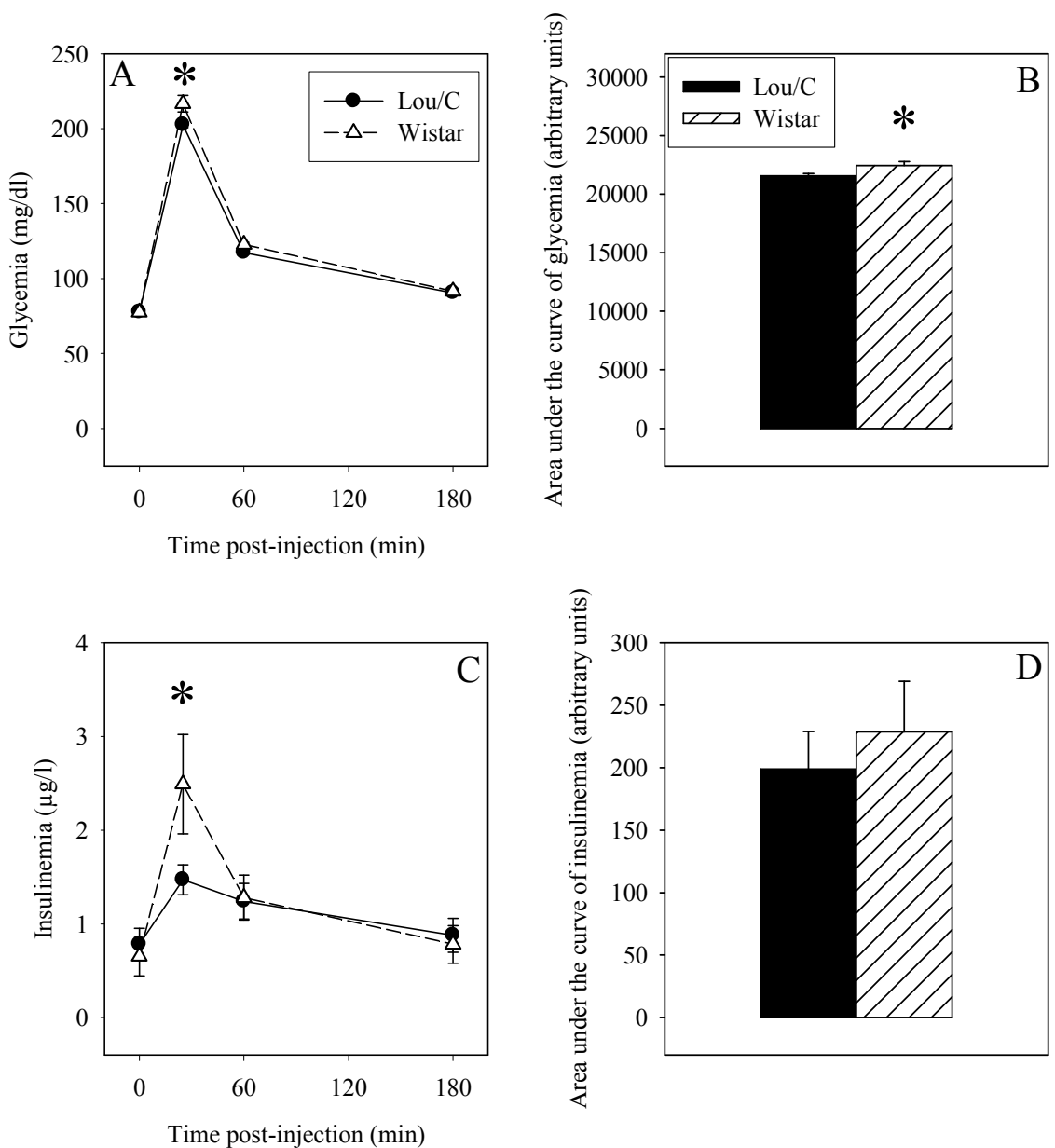

Figure 1: Influence of increased body weight and abdominal adiposity on systemic glucose tolerance. Panel A: time-evolution of glycaemia after glucose loading; Panel B: area under the curve for glycaemia; panel C: time-evolution of insulinemia after glucose loading; panel $\mathbf{D}$ : area under the curve for insulinemia. There were six experiments per group. *: Significantly different.

\begin{tabular}{|c|c|c|c|}
\hline & Lou/C & Wistar & Anova \\
\hline Systolic pressure (mmHg) & $135 \pm 3$ & $139 \pm 5$ & NS \\
\hline Diastolic pressure $(\mathrm{mmHg})$ & $12 \pm 0.5$ & $12 \pm 0.9$ & NS \\
\hline LVDP $(\mathrm{mmHg})$ & $123 \pm 3$ & $127 \pm 5$ & NS \\
\hline Heart rate (beats/min) & $311 \pm 9$ & $271 \pm 8$ & $p<0.01$ \\
\hline Rate pressure product (mHg/min) & $38 \pm 1$ & $34 \pm 1$ & $p<0.05$ \\
\hline Coronary flow $(\mathrm{ml} / \mathrm{min} / \mathrm{g})$ & $8.3 \pm 0.8$ & $6.6 \pm 0.3$ & $p<0.05$ \\
\hline Oxygen consumpt. ( $\mu$ moles/min/g) & $4.9 \pm 0.4$ & $4.1 \pm 0.2$ & NS \\
\hline Metabolic efficiency (mHg/umole) & $7.3 \pm 0.5$ & $5.4 \pm 0.2$ & $p<0.01$ \\
\hline Lactate release $(\mu \mathrm{moles} / \mathrm{min} / \mathrm{g})$ & $0.33 \pm 0.02$ & $0.47 \pm 0.04$ & $p<0.01$ \\
\hline Pyruvate release $(\mu \mathrm{moles} / \mathrm{min} / \mathrm{g})$ & $0.028 \pm 0.004$ & $0.034 \pm 0.004$ & NS \\
\hline Glycolytic flux ( $\mu$ moles/min/g) & $0,37 \pm 0.02$ & $0.51 \pm 0.04$ & $p<0.01$ \\
\hline Lactate to pyruvate ratio & $16 \pm 3$ & $16 \pm 3$ & NS \\
\hline $\begin{array}{l}\text { Ratio between released lactate and consumed oxygen } \\
\qquad(\mu \mathrm{moles} / \mu \mathrm{mole})\end{array}$ & $0.075 \pm 0.009$ & $0.119 \pm 0.011$ & $p<0.01$ \\
\hline
\end{tabular}

The number of experiments was 10 per group. LVDP: left ventricle developed pressure; Oxygen consumpt.: oxygen consumption; g: gram of heart weight; Anova: analysis of variance; NS: not significant.

Table 1: Ex- vivo cardiac function of Lou/C and Wistar rats under pre-ischemic conditions.

between the amount of lactate released and the quantity of oxygen consumed was noticeably higher $(+59 \%)$. This suggests increased participation of anaerobic glycolysis in energy production, which was associated with lower coronary flow $(-20 \%)$ and deteriorated cardiac metabolic efficiency (-26\%, RPP to oxygen consumption ratio).

\section{Myocardial NHE-1 and MCT- 1 contents}

To examine if the increased lactate production observed in Wistar rats under pre-ischemic conditions also occurred in-vivo, the ratio between the expression of the lactate extruders, MCT-1 and NHE- 
Citation: Vitiello D, Mourmoura E, Couturier K, Faure P, Dubouchaud H, et al. (2015) Increased Abdominal Adiposity Exacerbates Ex-Vivo Cardiac Reperfusion Injury through Augmented Mitochondrial Oxidative Stress. J Diabetes Metab 6: 614. doi:10.4172/2155-6156.1000614

Page 5 of 10

1, was evaluated. Figure 2 shows that Wistar rats displayed a higher myocardial ratio between MCT-1 and NHE-1 (+34\%).

\section{Ex-vivo cardiac function during ischemia/reperfusion}

During ischemia, contracture was greater in the Wistar phenotype $(+33 \%$ after 15 min of ischemia, Figure $3 \mathrm{~A})$. An additional increase in diastolic pressure that occurred after 5 min of reperfusion was also greater in the Wistar phenotype (890 vs. $697 \%$ of the pre-ischemic value). Despite a progressive decrease thereafter, diastolic pressure remained higher until the end of reperfusion $(+648$ and $+478 \%$ of the pre-ischemic value).

The LVDP (Figure 3B), heart rate (Figure 3C) and RPP (Figure $3 \mathrm{D}$ and $\mathrm{E}$ ) progressively recovered during reperfusion. Whatever the duration of reperfusion, the recovery of RPP was lower in the Wistar phenotype ( $-45 \%$ after 15 min of reperfusion, when the parameter was expressed as a percentage of pre-ischemic value): this was due to the slower recovery of LVDP and heart rate (-36 and $-37 \%$ after $15 \mathrm{~min}$ of reperfusion). Recoveries of coronary flow and oxygen consumption (Figure 4A and $\mathrm{B}$ ) were also lower in the Wistar phenotype (-24 and $-22 \%$ after $15 \mathrm{~min}$ of reperfusion). This phenomenon was associated with transitory lower lactate release in the coronary effluent $(-52 \%)$ and a lower lactate-flux to oxygen-consumption ratio (-30\%) after 5 min of reperfusion (Figure $4 \mathrm{C}$ and $\mathrm{D}$ ). This suggests that less intense anaerobic glycolysis occurred during the ischemic event. Despite these differences, the recovery of cardiac metabolic efficiency was not altered (data not shown).

\section{Mitochondrial oxidative phosphorylation}

As indicated by the activity of cardiac citrate synthase $(48.3 \pm 3.1 \mathrm{vs}$. $49.5 \pm 4.8 \mathrm{mU} / \mathrm{mg}$ of myocardial proteins), mitochondrial density was similar in the reperfused myocardium of both phenotypes.

Mitochondria were then extracted from the pre-ischemic and postischemic myocardium and oxidative phosphorylation was evaluated. The mitochondrial preparations were of high quality, as the RCR values were generally high, particularly in the calcium-rich media (Figure 5C).
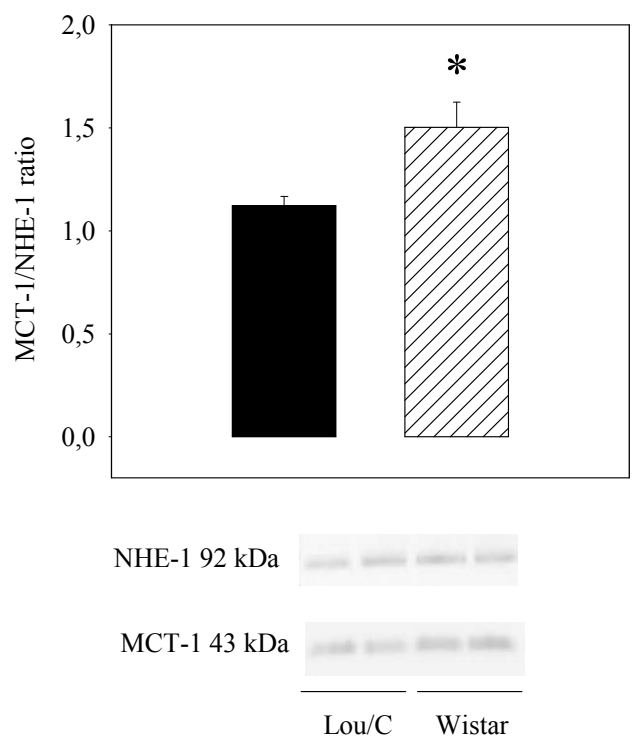

Figure 2: Influence of increased body weight and abdominal adiposity on the ratio between MCT-1 and NHE-1 in sarcolemmal membranes. There were ten experiments per group. *: Significantly different.
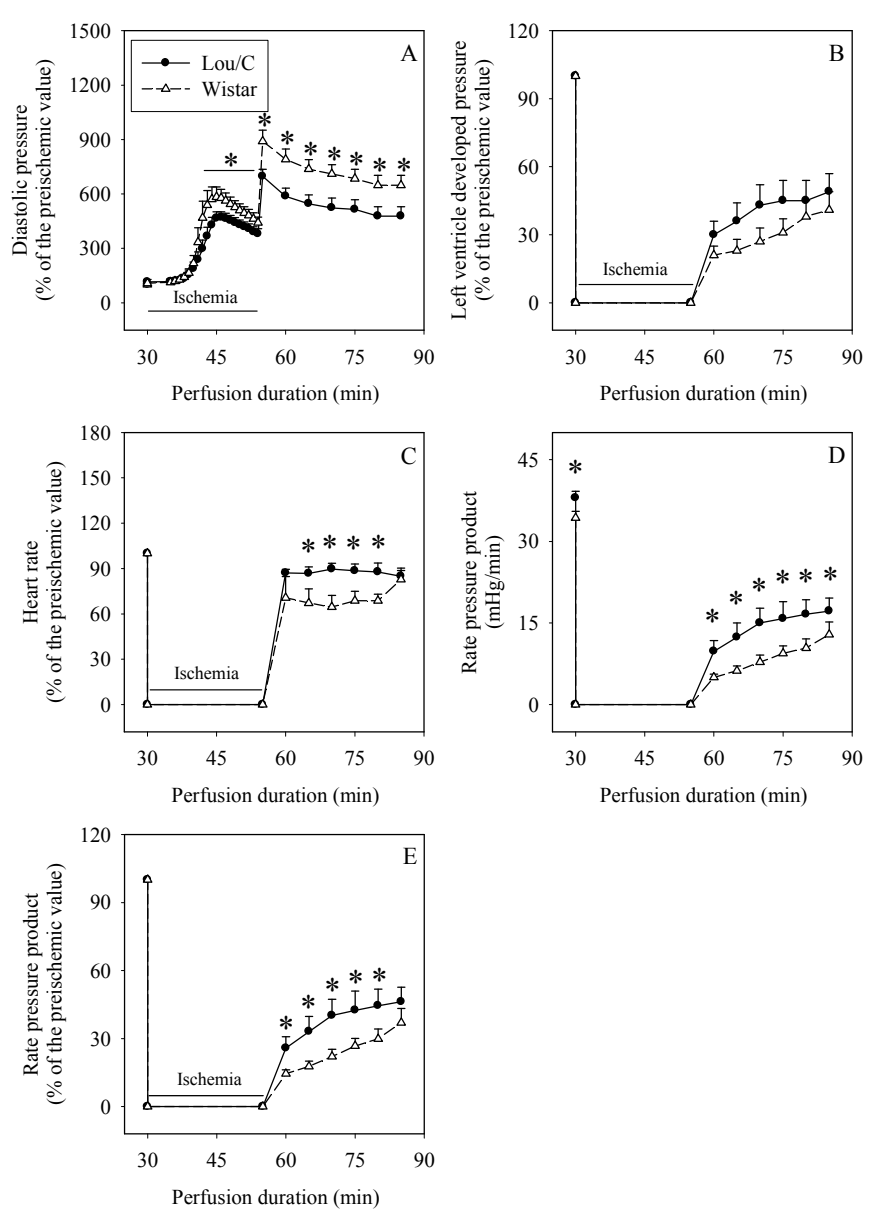

Figure 3: Influence of increased body weight and abdominal adiposity on diastolic pressure (panel A), left-ventricular pressure development (panel B), heart rate (panel $C$ ) and rate pressure product (panels $D$ and $E$, expressed in $\mathrm{mHg} / \mathrm{min}$ and $\%$ of pre-ischemic value) during ischemia/reperfusion. There were ten experiments per group. *: Significantly different.

In the mitochondria extracted from pre-ischemic myocardium, the presence of calcium increased the StIII. This was particularly noticeable in the Wistar phenotype $(+33 \%, p<0.05)$ when pyruvate/malate was used as the substrate. This did not occur with palmitoylcarnitine/ malate or a combination of the three substrates (data not shown). When mitochondria were extracted from post-ischemic myocardium, a calcium-induced stimulation of StIII was not observed.

The effects of phenotype on the parameters of oxidative phosphorylation were discrete. Differences were observed only when pyruvate/malate was used as a substrate. Indeed, the mitochondria from the pre-ischemic myocardium of Wistar rats displayed a higher RCR value $(+45 \%, p<0.05)$ when they were incubated in the presence of calcium. Moreover, the organelles isolated from the post-ischemic myocardium of the same phenotype showed a higher StIV $(+85$, $p<0.05)$ than those of Lou/C rats when they were incubated in calciumfree medium.

\section{Mitochondrial $\mathrm{H}_{2} \mathrm{O}_{2}$ release}

The results for $\mathrm{mH}_{2} \mathrm{O}_{2} \mathrm{r}$ are presented in Figure 6. For pre-ischemic mitochondria, phenotype did not modify $\mathrm{mH}_{2} \mathrm{O}_{2}$ r. For post-ischemic mitochondria incubated in a calcium-free medium, this was also generally the case. In contrast, when they were incubated in the 


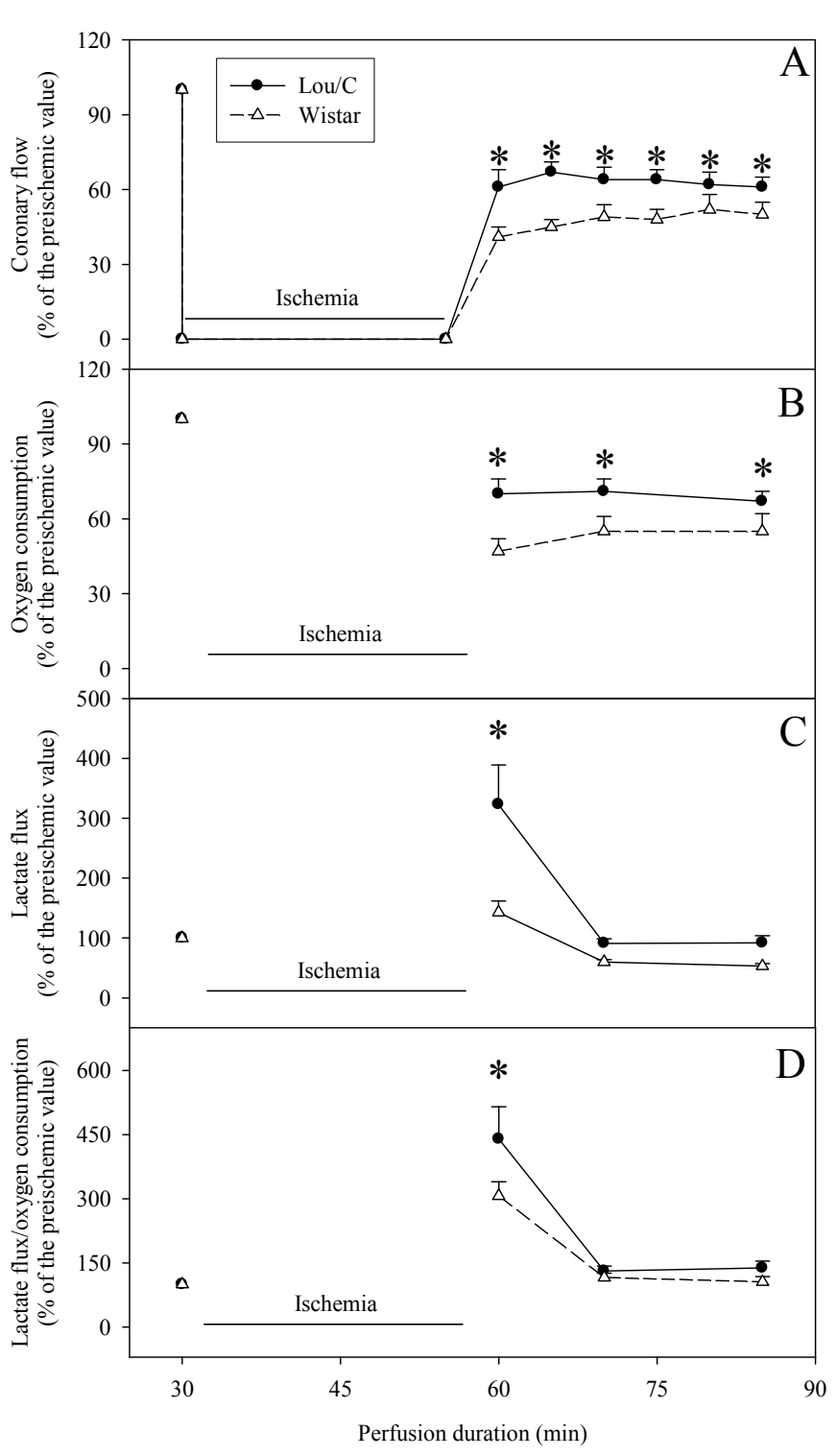

Figure 4: Influence of increased body weight and abdominal adiposity on coronary flow (panel A), myocardial oxygen consumption (panel B), lactate release in coronary effluents (panel $\mathrm{C}$ ) and ratio between lactate release and oxygen consumption (panel D) during post-ischemic reperfusion. There were ten experiments per group. *: Significantly different.

calcium-rich buffer, post-ischemic mitochondria from the Wistar phenotype displayed higher rates of $\mathrm{mH}_{2} \mathrm{O}_{2} \mathrm{r}$ whatever the substrate used $(+41,+25$ and $+41 \%$ with pyruvate, palmitoylcarnitine and pyruvate/palmitoylcarnitine, respectively).

The higher ROS of the post-ischemic mitochondria in the Wistar phenotype was also observed ex-vivo in the perfused heart. Indeed, the aconitase-to-fumarase ratio (Figure 7), which is inversely related to mitochondrial oxidative stress, was lower in the Wistar phenotype $(-23 \%)$. Higher oxidative stress was associated with higher activity of respiratory-chain complex II $(243 \pm 50$ vs. $137 \pm 11 \mathrm{mU} / \mathrm{U}$ of citrate synthase in the Lou/C phenotype, $+77 \%, p<0.05$ ) without any change in the activity of other respiratory chain complexes (data not shown).

\section{Discussion}

This study aimed to determine whether increased abdominal adiposity (IAA), at an early phase of systemic glucose intolerance, when augmented by intake of a low-fat diet equilibrated in lipids, could modify the recovery of mechanical function during post-ischemic reperfusion. We also evaluated if mitochondrial oxidative stress was involved, and assessed the reasons for the changes in ROS production.

Compared to Lou/C rats, the Wistar animals spontaneously ate more and had a much greater body weight. Furthermore, their body density and abdominal adiposity were augmented. This obviously indicates that Wistar rats had greater IAA compared to their Lou/C counterparts. These results agree with published data $[16,19,25]$.

\section{Strengths and weaknesses of the study}

The IAA observed in the Wistar phenotype was of interest for two reasons. First, it occurred in a range between thinness and low overcharge. Feeding Wistar rats with a Western-type high-fat diet leads to much greater abdominal adiposity (unpublished data). Thus, it is important to know whether gaining adiposity up to a moderate weight range modulates CSI. Secondly, our Wistar animals did eat the same low-fat food that was equilibrated in saturated $(24 \%)$, monounsaturated $(23 \%)$ and polyunsaturated (n-6, 48\%; $n-3,4.5 \%)$ fatty acids as Lou/C rats. This is far different to the classical Western diet, which is rich in saturated fats. Despite this, IAA triggered slight but significant systemic glucose intolerance. We do not know if such a disturbance in glucose metabolism also occurred in the heart, but the maintained lactate-to-pyruvate ratio of the coronary effluents indicates
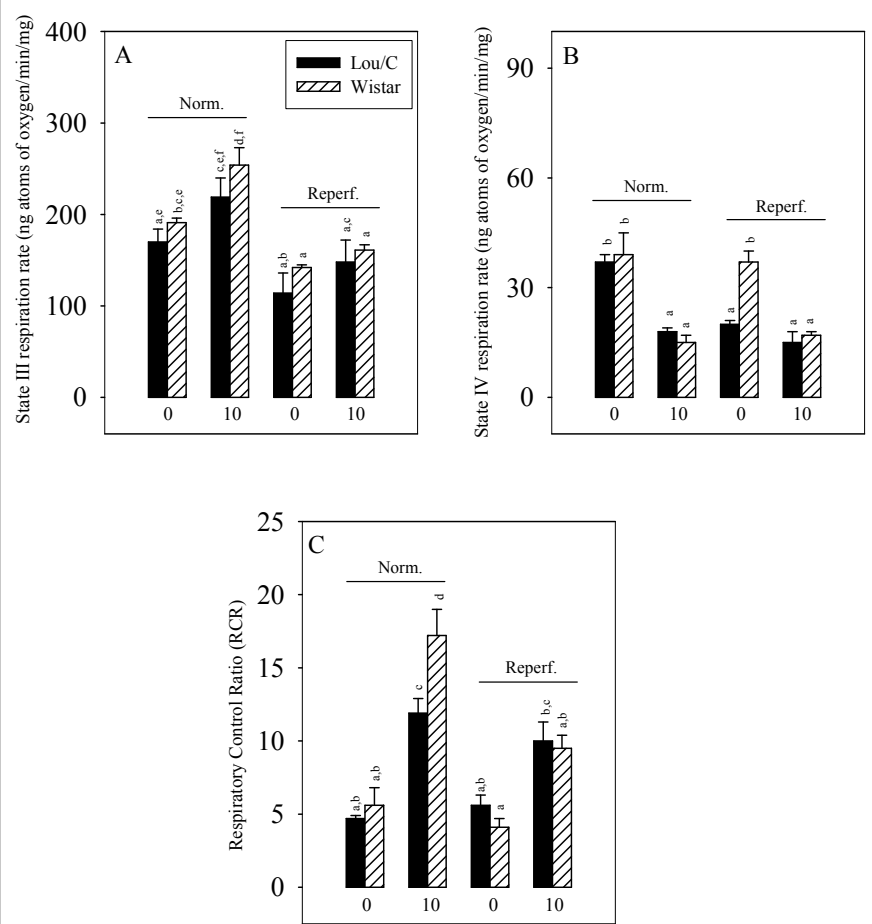

Figure 5: Influence of increased body weight and abdominal adiposity on the parameters of mitochondrial respiration (state-III respiration rate $=$ panels $\mathrm{A}$; state-IV respiration rate $=$ panels $\mathrm{B}$; respiratory-control ratio $=$ panels $\mathrm{C}$ ) when pyruvate/malate was given as substrates. There were six experiments per group. $a, b, c, d, e, f:$ the absence of a common letter between two means in the same panel indicates a significant difference. 

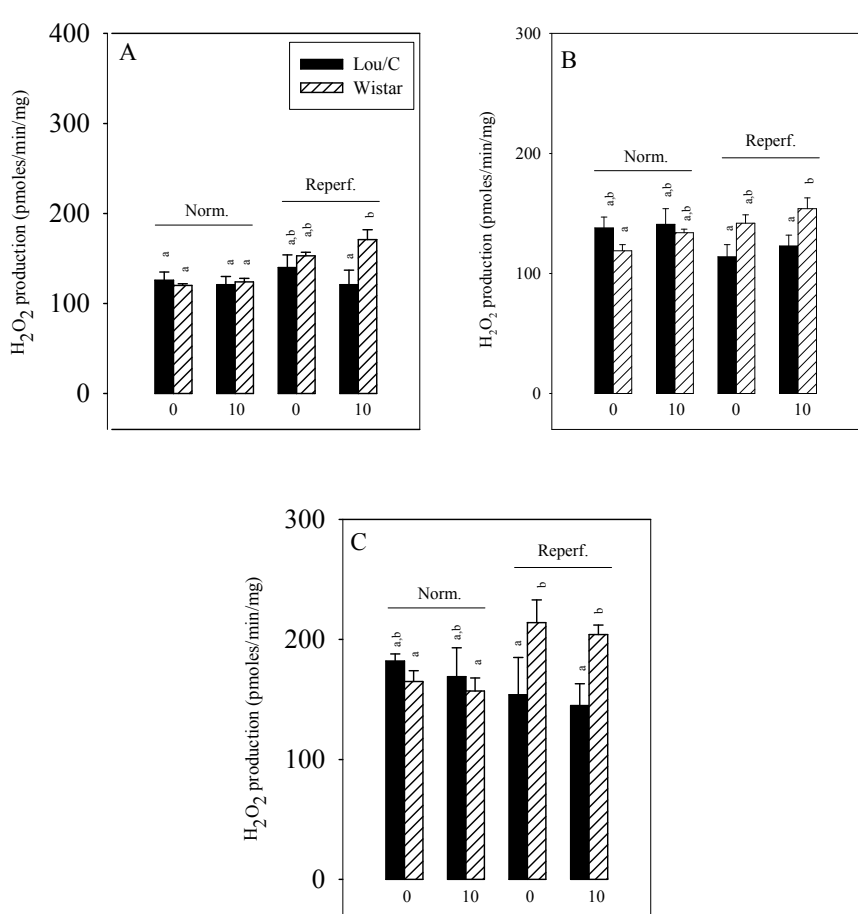

Figure 6: Influence of increased body weight and abdominal adiposity on mitochondrial basal hydrogen-peroxide release during state-II respiration when pyruvate/malate (panel A), palmitoylcarnitine/malate (panels B) and pyruvate/palmitoylcarnitine/malate (panels $C$ ) were given as substrates. There were six experiments per group. $a, b$ : the absence of a common letter between the two means in the same panel indicates a significant difference.

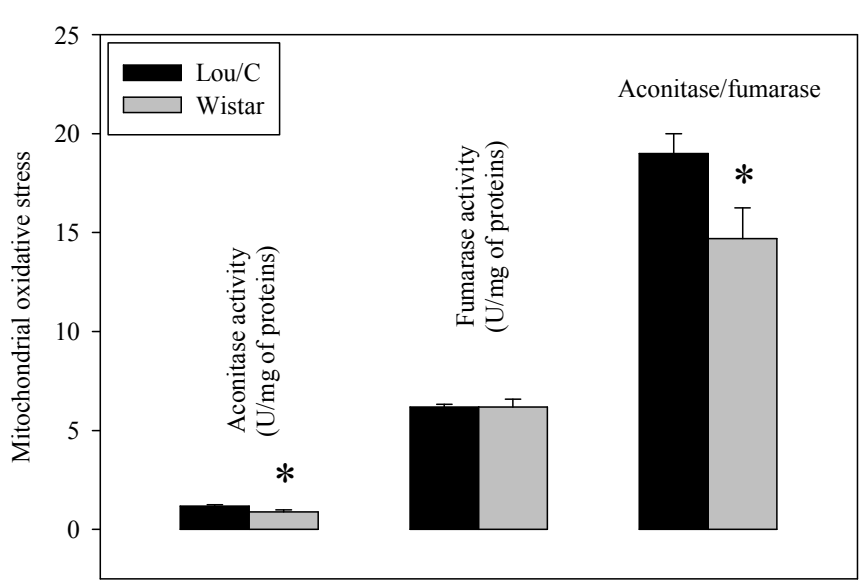

Figure 7: Influence of increased body weight and abdominal adiposity on the activities of aconitase and fumarase in post-ischemic cardiac homogenates, and on the aconitase-to-fumarase ratio. There were 10 experiments per group. *: Significantly different.

an unchanged cytosolic redox potential, and suggests the absence of cardiac glucose intolerance.

In our study, the effects of ischemia/reperfusion were evaluated exvivo in a model of an isolated perfused heart. The results could have been different in an in-vivo situation. Indeed, numerous systemic and nervous factors are modified by IAA. For instance, this is the case for two adipocytokines (adiponectin and leptin), whose systemic levels are oppositely modified by changes in abdominal adiposity. However, both are cardio-protective [46] and their integrated action cannot be modified.

Our ex-vivo model could also differ from an in-vivo situation because of other factors. First, our perfusion fluid was devoid of insulin. Du Toit et al. [6] have shown that adding insulin to the perfusion fluid of hearts derived from Western diet-fed rats abolished the difference previously observed in the absence of this hormone. Second, the same authors [6] have also demonstrated that adding free fatty acids to the perfusion fluid strongly increased the CSI, but abolished the previously observed differences. Yet, our perfusion fluid contained only glucose as an energy substrate, which is usually sufficient to allow the heart to develop high mechanical activity, even in working mode $[46,47]$. Other researchers have supplied their isolated hearts with a mixture of glucose, lactate, pyruvate and palmitate $[13,14]$. Although this precaution could improve cardiac energy metabolism, it cannot mimic the in-vivo situation in which the systemic NEFA level could be modified by IAA [48]. Thirdly, IAA is associated with low-grade inflammation [49], which could also have played a role. In spite of these environmental differences, several studies [2-9] have shown that the response to ischemia/reperfusion was similar in both in-vivo and exvivo contexts.

\section{Cardiac function with pre-ischemic conditions}

In our study, Wistar rats displayed a lower RPP, which was caused by a deteriorated heart rate. This confirms the effect of IAA in different ex-vivo models, including in Western diet-fed rats [5,7], ZDF animals $[13,14,45]$ and mice subjected to ON [11]. This phenomenon was associated with lower coronary flow and cardiac metabolic efficiency. The IAA-induced decrease in metabolic efficiency has already been demonstrated in Wistar rats fed a high-fat diet compared to animals of the same phenotype fed a control chow [50]. It is thought to be caused by a decreased mitochondrial ATP:O ratio, possibly related to increased uncoupling of protein-3 expression [50]. It validates the hypothesis previously assumed [20] and suggests that the Lou/C rat displayed a higher metabolic efficiency. Moreover, we have shown, for the first time that Wistar rats displayed higher cardiac lactate production and a higher ratio between the amount of lactate released and oxygen consumed when compared to Lou/C rats. This reflects the stimulation of anaerobic glycolysis, which occurs when oxygen supply and aerobic ATP production are insufficient to meet energy needs. This allows anaerobic production of ATP in the cytosol through reoxidation of glycolytic NADH. Because it occurs during disequilibrium between oxygen supply and energy needs, such as hyp(an)oxia, it clearly indicates that, as already reported [51], coronary flow is a limiting factor during ex-vivo cardiac perfusion in the Wistar rat. Furthermore, we observed that the content of MCT-1, the monocarboxylate carrier that allows lactate release from the cytosol, was higher in the myocardium of Wistar rats. This adaptation strongly suggests that lactate release from the heart as well as anaerobic glycolysis were constantly stimulated in the whole animal, and that coronary flow was insufficient to meet the energy needs in the in-vivo situation. The regulation of several mitochondrial enzymes could also be modified by IAA-induced changes to the environment. Cole et al. [50] evoked augmented cardiac uncoupling of protein-3 expression, which was then responsible for reduced myocardial metabolic efficiency.

In our study, interesting results with isolated mitochondria extracted from the pre-ischemic myocardium of the two phenotypes were obtained regarding the effect of calcium. In general, the addition of calcium into the mitochondrial preparation increased RCR values through the augmentation of state-III and a decrease in 
Citation: Vitiello D, Mourmoura E, Couturier K, Faure P, Dubouchaud H, et al. (2015) Increased Abdominal Adiposity Exacerbates Ex-Vivo Cardiac Reperfusion Injury through Augmented Mitochondrial Oxidative Stress. J Diabetes Metab 6: 614. doi:10.4172/2155-6156.1000614

Page 8 of 10

state-IV respiration rates. Inter-strain differences were caused by changes to state-III respiration rates. In the IAA mitochondria, the calcium-induced stimulation of state-III respiration rate [52] was observed with pyruvate, but not with palmitoylcarnitine. This agrees perfectly with the increased glucose-oxidation rate observed in the hearts of these animals when RPP is augmented [53]. In contrast, in the lean rat, the stimulatory effect of calcium was observed more with palmitoylcarnitine. This reveals the known preference of this phenotype for lipid substrates $[21,25]$. This phenomenon could have occurred in our ex-vivo preparation because, although our perfusion fluid contained only glucose as a substrate, cardiac lipid stores could account for the $\beta$-oxidation activity. In our study, we did not evaluate myocardial $\beta$-oxidation of the two strains. However, this metabolic pathway could contribute to the changes in cardiac metabolic efficiency observed in both the strains.

\section{Cardiac function during ischemia/reperfusion}

The IAA rats demonstrated lower RPP recovery during reperfusion compared to the lean phenotype. This agrees well with results obtained ex-vivo [5-7] and in-vivo $[4,8,9]$ in rats fed a HF diet. However, this is in total opposition with the results from ZDF rats $[13,14]$ and SMC-fed animals $[2,3]$. Yet, the two last situations are associated with increased blood glucose, whereas the increased chow intake was not in our study. Thus, it seems that high blood-glucose affords cardio-protection, whereas high fat intake is toxic. ON $[11,12]$ and increased food intake in the Wistar compared to the Lou/C rat resembles the HF-diet situation.

As evidenced by the lower recovery of oxygen consumption, the IAA-related cardio-toxicity can be partly explained by abnormalities in mitochondrial metabolism. One of the most striking findings of our study was the higher activity of respiratory-chain complex II in the reperfused myocardium of IAA rats compared to the lean phenotype. Ischemia/reperfusion is known to inhibit complex II [54]. Initially, this was attributed to increased oxidative stress. However, a recent study [55] has reported the huge influence of intracellular $\mathrm{pH}$. With acidosis, a situation that is known to occur during ischemia/reperfusion, the SDHA and SDHB subunits of succinate dehydrogenase split-up from succinate-ubiquinone-reductase, which results in decreased activity of the enzyme. It is possible that reperfusion did not allow total restoration of $\mathrm{pH}$ in the Lou/C rat, whereas neutralization was achieved in the Wistar phenotype. This could occur through the increased content of MCT-1, as observed in the Wistar strain. Indeed, this transport system co-expulses mono-carboxylates and protons out of the cell.

The maintained complex-II activity had a strong influence on $\mathrm{mH}_{2} \mathrm{O}_{2} \mathrm{r}$ by increasing that parameter in IAA rats when calcium was added to the respiration medium. Calcium is known to trigger the accumulation of the Krebs-cycle intermediate, succinate, in the mitochondrial environment [56]. Succinate enters the respiratory chain at the level of complex II and induces a huge $\mathrm{mH}_{2} \mathrm{O}_{2} \mathrm{r}$ at the complex-I level, via a reverse electron flux [40]. Since the activity of complex II was higher in IAA, the $\mathrm{mH}_{2} \mathrm{O}_{2} \mathrm{r}$ was thus higher. Interestingly, the higher mitochondrial oxidative stress measured in-vitro was also observed exvivo: we found a reduced aconitase-to-fumarase ratio. Such oxidative stress is known to decrease activity in the Krebs cycle [37], which probably explains the increase in IAA-induced CSI through a reduced rate of energy synthesis.

Recently, we have shown that the transition from 3 to 6 months of age increases ischemia-induced damage but that, until the age of 18 months, these consequences did not worsen [57]. This was attributed to the surprising maintenance of complex-II activity and increased
ROS production [40]. Preliminary results from our laboratory show a huge increase in IAA between 3 and 6 months of age and relative stabilization thereafter [58]. These results, associated with those of the present study, suggest that the accumulation of abdominal fat observed between 3 and 6 months is responsible for the increased CSI.

\section{Impact on human nutrition}

IAA and/or a slight increase in systemic glucose intolerance has a deleterious effect on cardiac function during ischemia/reperfusion by favoring the maintenance of complex II activity and increasing mitochondrial oxidative stress. Utilization of a complex-II inhibitor could thus afford cardio-protection in overweight and obese individuals.

\section{Acknowledgements}

This work was supported by the Institut National de la Recherche Agronomique (INRA), National Institute of Health and Medical Research (INSERM) and the Joseph Fourier University. The authors thank Christophe Cottet for carefully editing the manuscript and Cindy Tellier for animal care.

\section{References}

1. Bales CW, Buhr GT (2009) Body mass trajectory, energy balance, and weight loss as determinants of health and mortality in older adults. Obes Facts 2: 171 178.

2. Donner D, Headrick JP, Peart JN, du Toit EF (2013) Obesity improves myocardial ischaemic tolerance and RISK signalling in insulin-insensitive rats. Dis Model Mech 6: 457-466.

3. Huisamen B, Dietrich D, Bezuidenhout N, Lopes J, Flepisi B, et al. (2012) Early cardiovascular changes occurring in diet-induced, obese insulin-resistant rats. Mol Cell Biochem 368: 37-45.

4. Aubin MC, Cardin S, Comtois P, Clement R, Gosselin H, et al. (2010) A high-fat diet increases risk of ventricular arrhythmia in female rats: enhanced arrhythmic risk in the absence of obesity or hyperlipidemia. J Appl Physiol 108: 933-940.

5. du Toit EF, Nabben M, Lochner A (2005) A potential role for angiotensin II in obesity induced cardiac hypertrophy and ischaemic/reperfusion injury. Basic Res Cardiol 100: 346-354.

6. du Toit EF, Smith W, Muller C, Strijdom H, Stouthammer B, et al. (2008) Myocardial susceptibility to ischemic-reperfusion injury in a prediabetic model of dietary-induced obesity. Am J Physiol Heart Circ Physiol 294: 2336-2343.

7. Essop MF, Anna Chan WY, Valle A, Garcia-Palmer FJ, Du Toit EF (2009) Impaired contractile function and mitochondrial respiratory capacity in response to oxygen deprivation in a rat model of pre-diabetes. Acta Physiol (Oxf) 197: 289-296.

8. Huang JV, Lu L, Ye S, Bergman BC, Sparagna GC, et al. (2013) Impaired contractile recovery after low-flow myocardial ischemia in a porcine model of metabolic syndrome. Am J Physiol Heart Circ Physiol 304: 861-873.

9. Thakker GD, Frangogiannis NG, Bujak M, Zymek P, Gaubatz JW, et al. (2006) Effects of diet-induced obesity on inflammation and remodeling after myocardial infarction. Am J Physiol Heart Circ Physiol 291: H2504-2514.

10. Calvert JW, Lefer DJ, Gundewar S, Poston L, Coetzee WA (2009) Developmental programming resulting from maternal obesity in mice: effects on myocardial ischaemia-reperfusion injury. Exp Physiol 94: 805-814.

11. Granado M, Fernandez N, Monge L, Figueras JC, Carreno-Tarragona G, et al. (2013) Effects of coronary ischemia-reperfusion in a rat model of early overnutrition. Role of angiotensin receptors. PLoS One 8: e54984.

12. Habbout A, Delemasure S, Goirand F, Guilland JC, Chabod F, et al. (2012) Postnatal overfeeding in rats leads to moderate overweight and to cardiometabolic and oxidative alterations in adulthood. Biochimie 94: 117-124.

13. Wang P, Chatham JC (2004) Onset of diabetes in Zucker diabetic fatty (ZDF) rats leads to improved recovery of function after ischemia in the isolated perfused heart. Am J Physiol Endocrinol Metab 286: 725-736.

14. Wang P, Lloyd SG, Zeng H, Bonen A, Chatham JC (2005) Impact of altered substrate utilization on cardiac function in isolated hearts from Zucker diabetic fatty rats. Am J Physiol Heart Circ Physiol 288: 2102-2110. 
Citation: Vitiello D, Mourmoura E, Couturier K, Faure P, Dubouchaud H, et al. (2015) Increased Abdominal Adiposity Exacerbates Ex-Vivo Cardiac Reperfusion Injury through Augmented Mitochondrial Oxidative Stress. J Diabetes Metab 6: 614. doi:10.4172/2155-6156.1000614

15. Sidell RJ, Cole MA, Draper NJ, Desrois M, Buckingham RE, et al. (2002) Thiazolidinedione treatment normalizes insulin resistance and ischemic injury in the zucker Fatty rat heart. Diabetes 51: 1110-1117.

16. Garait B, Couturier K, Servais S, Letexier D, Perrin D, et al. (2005) Fat intake reverses the beneficial effects of low caloric intake on skeletal muscle mitochondrial $\mathrm{H}(2) \mathrm{O}(2)$ production. Free Radic Biol Med 39: 1249-1261.

17. Peltier S, Novel-Chate V, Malaisse WJ, Molnar A, Leverve XM, et al. (2007) Cardioprotective effect of spontaneous activity. Int J Sports Med 28: 987-993.

18. Taleux N, De Potter I, Deransart C, Lacraz G, Favier R, et al. (2008) Lack of starvation-induced activation of AMP-activated protein kinase in the hypothalamus of the Lou/C rats resistant to obesity. Int J Obes (Lond) 32: 639647.

19. Veyrat-Durebex C, Montet X, Vinciguerra M, Gjinovci A, Meda P, et al. (2009) The Lou/C rat: a model of spontaneous food restriction associated with improved insulin sensitivity and decreased lipid storage in adipose tissue. Am J Physiol Endocrinol Metab 296: 1120-1132.

20. Taleux N, Guigas B, Dubouchaud H, Moreno M, Weitzel JM, et al. (2009) High expression of thyroid hormone receptors and mitochondrial glycerol-3phosphate dehydrogenase in the liver is linked to enhanced fatty acid oxidation in Lou/C, a rat strain resistant to obesity. J Biol Chem 284: 4308-4316.

21. Veyrat-Durebex $C$, Poher AL, Caillon A, Montet $X$, Rohner-Jeanrenaud $F$ (2011) Alterations in lipid metabolism and thermogenesis with emergence of brown adipocytes in white adipose tissue in diet-induced obesity-resistan Lou/C rats. Am J Physiol Endocrinol Metab 300: 1146-1157.

22. Couturier K, Servais S, Koubi H, Sempore B, Cottet-Emard JM, et al. (2004) Metabolic and hormonal responses to exercise in the anti-obese Lou/C rats. Int J Obes Relat Metab Disord 28: 972-978.

23. Mitchell SE, Nogueiras R, Rance K, Rayner DV, Wood S, et al. (2006) Circulating hormones and hypothalamic energy balance: regulatory gene expression in the Lou/C and Wistar rats. J Endocrinol 190: 571-579.

24. Marissal-Arvy N, Gaumont A, Langlois A, Dabertrand F, Bouchecareilh M, et al. (2007) Strain differences in hypothalamic pituitary adrenocortical axis function and adipogenic effects of corticosterone in rats. J Endocrinol 195: 473-484.

25. Marissal-Arvy N, Langlois A, Tridon C, Mormede P (2011) Functional variability in corticosteroid receptors is a major component of strain differences in fat deposition and metabolic consequences of enriched diets in rat. Metabolism 60: 706-719.

26. Soulage C, Zarrouki B, Soares AF, Lagarde M, Geloen A (2008) Lou/C obesityresistant rat exhibits hyperactivity, hypermetabolism, alterations in white adipose tissue cellularity, and lipid tissue profiles. Endocrinology 149: 615-625.

27. Lacraz G, Couturier K, Taleux N, Servais S, Sibille B, et al. (2008) Liver mitochondrial properties from the obesity-resistant Lou/C rat. Int J Obes (Lond) 32: $629-638$

28. Tadic M, Ivanovic B, Cuspidi C (2013) What do we currently know about metabolic syndrome and atrial fibrillation? Clin Cardiol 36: 654-662.

29. Guerra F, Rey de Viñas JL, Ortega R (1981) [Effect of sodium and potassium on cardiac infarct in rats]. Arch Inst Cardiol Mex 51: 3-12.

30. Demaison L, Moreau D, Vergely-Vandriesse C, Gregoire S, Degois M, et al (2001) Effects of dietary polyunsaturated fatty acids and hepatic steatosis on the functioning of isolated working rat heart under normoxic conditions and during post-ischemic reperfusion. Mol Cell Biochem 224: 103-116.

31. Van Bilsen M, van der Vusse GJ, Coumans WA, de Groot MJ, Willemsen PH, et al. (1989) Degradation of adenine nucleotides in ischemic and reperfused rat heart. Am J Physiol 257: H47-54.

32. Robergs RA, Ghiasvand F, Parker D (2004) Biochemistry of exercise-induced metabolic acidosis. Am J Physiol Regul Integr Comp Physiol 287: R502-516.

33. Dong JW, Zhu HF, Zhou ZN (2003) Inhibitors of $\mathrm{Na}+/ \mathrm{H}+$ and $\mathrm{Na}+/ \mathrm{Ca} 2+$ exchange depress intracellular calcium elevation induced by ischemia/ reperfusion in rat cardiac myocytes. Sheng Li Xue Bao 55: 245-250.

34. Muscari A, De Pascalis S, Cenni A, Ludovico C, Castaldini N, et al. (2008) Determinants of mean platelet volume (MPV) in an elderly population: relevance of body fat, blood glucose and ischaemic electrocardiographic changes. Thromb Haemost 99: 1079-1084.

35. Miyata H, Lakatta EG, Stern MD, Silverman HS (1992) Relation of mitochondrial and cytosolic free calcium to cardiac myocyte recovery after exposure to anoxia. Circ Res 71: 605-613.

36. Martin C, Dubouchaud H, Mosoni L, Chardigny JM, Oudot A, et al. (2007) Abnormalities of mitochondrial functioning can partly explain the metabolic disorders encountered in sarcopenic gastrocnemius. Aging Cell 6: 165-177.

37. Li J, Gao X, Qian M, Eaton JW (2004) Mitochondrial metabolism underlies hyperoxic cell damage. Free Radic Biol Med 36: 1460-1470.

38. Alves MG, Oliveira PJ, Carvalho RA (2009) Mitochondrial preservation in Celsior versus histidine buffer solution during cardiac ischemia and reperfusion. Cardiovasc Toxicol 9: 185-193.

39. Gnaiger E (2001) Bioenergetics at low oxygen: dependence of respiration and phosphorylation on oxygen and adenosine diphosphate supply. Respir Physio 128: $277-297$.

40. Mourmoura E, Leguen M, Dubouchaud H, Couturier K, Vitiello D, et al. (2011) Middle age aggravates myocardial ischemia through surprising upholding of complex II activity, oxidative stress, and reduced coronary perfusion. Age (Dordr) 33: 321-336

41. Dubouchaud H, Butterfield GE, Wolfel EE, Bergman BC, Brooks GA (2000) Endurance training, expression, and physiology of LDH, MCT1, and MCT4 in human skeletal muscle. Am J Physiol Endocrinol Metab 278: E571-579.

42. Bergmeyer H (1974) Methods of enzymatic analysis. HU Bergmeyer, editor ed: London: Academic Press.

43. Faloona GR, Srere PA (1969) Escherichia coli citrate synthase. Purification and the effect of potassium on some properties. Biochemistry 8: 4497-4503.

44. Gardner PR, Nguyen DD, White CW (1994) Aconitase is a sensitive and critical target of oxygen poisoning in cultured mammalian cells and in rat lungs. Proc Natl Acad Sci U S A 91: 12248-12252.

45. Mourmoura E, Vial G, Laillet B, Rigaudiere JP, Hininger-Favier I, et al. (2013) Preserved endothelium-dependent dilatation of the coronary microvasculature at the early phase of diabetes mellitus despite the increased oxidative stress and depressed cardiac mechanical function ex vivo. Cardiovasc Diabetol 12 49.

46. Smith CC, Mocanu MM, Davidson SM, Wynne AM, Simpkin JC, et al. (2006) Leptin, the obesity-associated hormone, exhibits direct cardioprotective effects. Br J Pharmacol 149: 5-13.

47. Rochette L, Didier JP, Moreau D, Bralet J (1980) Effect of substrate on release of myocardial norepinephrine and ventricular arrhythmias following reperfusion of the ischemic isolated working rat heart. J Cardiovasc Pharmacol 2: 267-279.

48. Capurso C, Capurso A (2012) From excess adiposity to insulin resistance: the role of free fatty acids. Vascul Pharmacol 57: 91-97.

49. Di Lorenzo C, Dell'agli M, Colombo E, Sangiovanni E, Restani P (2013) Metabolic syndrome and inflammation: a critical review of in vitro and clinical approaches for benefit assessment of plant food supplements. Evid Based Complement Alternat Med 2013: 782461.

50. Cole MA, Murray AJ, Cochlin LE, Heather LC, McAleese S, et al. (2011) A high fat diet increases mitochondrial fatty acid oxidation and uncoupling to decrease efficiency in rat heart. Basic Res Cardiol 106: 447-457.

51. Poizat C, Keriel C, Cuchet P (1994) Is oxygen supply sufficient to induce normoxic conditions in isolated rat heart? Basic Res Cardiol 89: 535-544.

52. Traaseth N, Elfering S, Solien J, Haynes V, Giulivi C (2004) Role of calcium signaling in the activation of mitochondrial nitric oxide synthase and citric acid cycle. Biochim Biophys Acta 1658: 64-71.

53. Neely JR, Whitmer M, Mochizuki S (1976) Effects of mechanical activity and hormones on myocardial glucose and fatty acid utilization. Circ Res 38: I22-30.

54. Chen YR, Chen CL, Pfeiffer DR, Zweier JL (2007) Mitochondrial complex II in the post-ischemic heart: oxidative injury and the role of protein S-glutathionylation. J Biol Chem 282: 32640-32654.

55. Grimm S (2013) Respiratory chain complex II as general sensor for apoptosis Biochim Biophys Acta 1827: 565-572.

56. Sentex E, Laurent A, Martine L, Gregoire S, Rochette L, Demaison L (1999) Calcium- and ADP-magnesium-induced respiratory uncoupling in isolated cardiac mitochondria: influence of cyclosporin A. Mol Cell Biochem 202: 73-84.

57. Oudot A, Martin C, Busseuil D, Vergely C, Demaison L, Rochette L (2006) 
Citation: Vitiello D, Mourmoura E, Couturier K, Faure P, Dubouchaud H, et al. (2015) Increased Abdominal Adiposity Exacerbates Ex-Vivo Cardiac Reperfusion Injury through Augmented Mitochondrial Oxidative Stress. J Diabetes Metab 6: 614. doi:10.4172/2155-6156.1000614

Page 10 of 10

NADPH oxidases are in part responsible for increased cardiovascular superoxide production during aging. Free Radic Biol Med 40: 2214-2222.

58. Mourmoura E, Couturier K, Hininger-Favier I, Malpuech-Brugere C, Azarnoush
$\mathrm{K}$, et al. (2014) Functional changes of the coronary microvasculature with aging regarding glucose tolerance, energy metabolism, and oxidative stress. Age (Dordr) 36: 9670 . 